\title{
A Novel Knee Orthosis in the Treatment of Patellofemoral Pain Syndrome
}

\author{
Michael J. Khadavi, Y. T. Chen, Michael Fredericson \\ Carondelet Orthopaedic Surgeons and Sports Medicine, Overland Park, KS, USA \\ Email: mjkhadavi@gmail.com
}

Received 23 March 2015; accepted 22 May 2015; published 25 May 2015

Copyright (C) 2015 by authors and Scientific Research Publishing Inc.

This work is licensed under the Creative Commons Attribution International License (CC BY). http://creativecommons.org/licenses/by/4.0/

(c) (i) Open Access

\section{Abstract}

Context: Patellofemoral Pain (PFPS) accounts for up to $25 \%$ of knee injuries in sports medicine clinics, with up to $91 \%$ of symptoms unresolved after conservative treatment at 5 years. The variability of response to treatment reflects its multi-factorial biomechanical etiologies. Bracing has been utilized to modify patellofemoral kinematics, generally by increasing patellofemoral contact area. The DJO Reaction orthosis is unique in its shock-absorbing elastomeric design, which is created to dissipate peak stress and enhance patellar tracking. Objective: To assess whether the DJO Reaction Brace reduces pain and improves functional outcomes in patients with chronic PFPS. Design: Cohort Series. Setting: Academic Sports Medicine Clinic. Patients: Twenty-two individuals between 18 and 40 years old with chronic patellofemoral pain have failed conservative treatment. Intervention: DJO Reaction Brace. Main Outcome Measures: Kujala Anterior Knee Pain Scale, Knee Injury and Osteoarthritis Outcome Score. Results: Seven males and 10 females with an age range of 19 - 39 years old complete the study. At an average follow-up time of 55 days, the Kujala score significantly improves by $9.8 \%$, and KOOS Scores increase by the following statistically significant amounts: symptom $3.2 \%$, pain $10.7 \%$, sports and recreation $12.9 \%$, quality of life $20.2 \%$. Conclusion: The DJO Reaction orthosis reduces knee pain, increases function, and enhances quality of life with individuals with PFPS and is effective in the conservative care of patellofemoral pain syndrome.

\section{Keywords}

Knee Orthosis, Knee Injuries, Treatment, Patellofemoral Kinematics

\section{Introduction}

Patellofemoral pain syndrome (PFPS) is a condition characterized by insidious onset anterior knee pain that is 
magnified in the setting of increased compressive forces to the patellofemoral joint, such as squatting, ascending or descending stairs, and prolonged sitting [1]. PFPS is remarkably common, accounting for up to $25 \%$ of all knee injuries presenting to sports medicine clinics [2]. The precise etiology, however, remains poorly defined. Current understanding supports a multifactorial basis in which a diverse array of contributing factors result in excessive patellofemoral joint stress.

Conservative care for PFPS has emphasized: strengthening the quadriceps and hip abductors; improving flexibility of the rectus femoris, hamstrings, calves, and hip flexors; decreasing excessive pronation at the foot through foot orthoses; non-steroidal anti-inflammatory medications; and taping or bracing to alter patellofemoral joint mechanics [3]. However, mounting evidence suggests that despite conservative care, PFPS persists in $80 \%$ 91\% of individuals at 5-year follow-up [4]. The chronicity and variability of response to treatment likely reflects the diverse and multifactorial mechanisms that lead to increased patellofemoral joint stress and resultant pain and disability. Moreover, at this time, individual responses to specific conservative strategies in isolation are unpredictable [5].

Thus, to address these factors, bracing has been explored for its role in patellar stabilization and management of PFPS. Bracing has been shown to modify patellofemoral kinematics [6] [7]. Medially, directed patellofemoral stabilization braces and, to a lesser degree simple knee support sleeves, contribute to reducing lateral translation of the patella. Medially directed patellofemoral stabilization braces may also reduce patellar tilt. These alterations are more apparent in a subset of individuals who have baseline abnormal kinematics when compared to controls [6]. However, it is recognized that the impact of braces on patellar lateralization and tilt is limited and alignment is not restored to normal with bracing alone [6] [7]. Patellofemoral contact area, on the other hand, does appear to increase substantially with the application of a medially directed brace and may be a driving force in associated pain reduction [7].

There are few prospective randomized clinical trials evaluating the impact of bracing and the clinical effect of knee braces on patellofemoral pain has shown varying results. Immediate pain reduction following application of a brace has been reported [7]. Longer-term benefits in a small population have also been demonstrated [8]. Arazpour, et al., find a nearly 60\% reduction in pain with the use of bracing and additionally ascertained gains in walking speed and step length [8]. Despite this, others have failed to uncover a therapeutic benefit to bracing [9]. This may represent inappropriate patient selection (normal patellar kinematics) or variability in the type of brace employed.

An ideal design for a knee orthosis may shift peak loads away from the painful lateral patellofemoral joint and dissipate the peak stress to allow improved tracking, while also enhancing proprioceptive and neurosensory feedback. Furthermore, identification of the subpopulation of patients with PFPS who attain the greatest benefit from a knee orthosis would enhance clinical efficacyand outcomes. The knee orthosis under current study is the DJO Reaction Brace (see Figures 1-3). It is unique in its shock-absorbing elastomeric design, which is created to accomplish the aforementioned biomechanical objectives.

The primary objective of this study is to assess whether the DJO Reaction Brace results in a reduction in pain and improve functional outcomes in patients with chronic PFPS. Our primary hypothesis is that the DJO Reaction Brace will result in reduction of pain during common functional tasks in patients with chronic PFPS who have failed conservative care.

\section{Methods}

\subsection{Design: Cohort}

Patients: Prior to the study, we obtained internal review board (IRB) approval. All patients signed a consent form before participating in this study. Between October 2012 and April 2015, we recruited twenty-two patients between the ages of 18 and 40 at an academic sports medicine clinic with chronic patellofemoral pain syndrome. This condition is defined as anterior knee pain for at least 3 months that is reproducible during at least two of the following tasks: kneeling, squatting, prolonged sitting, walking up/down stairs, squatting, or isometric quadriceps contraction. We selected an upper age limit of 40 to reduce the likelihood of recruiting patients with degenerative joint disease. Exclusion criteria included a history of patellar subluxation or dislocation, knee ligament instability, lower extremity muscle or tendon rupture, patellar tendonitis or bursitis, lower extremity trauma, degenerative joint disease, neurologic disease that would affect jogging or squatting, or knee surgery.

Procedures: All subjects were then fitted with the DJO Reaction Brace and instructed to wear this for all 


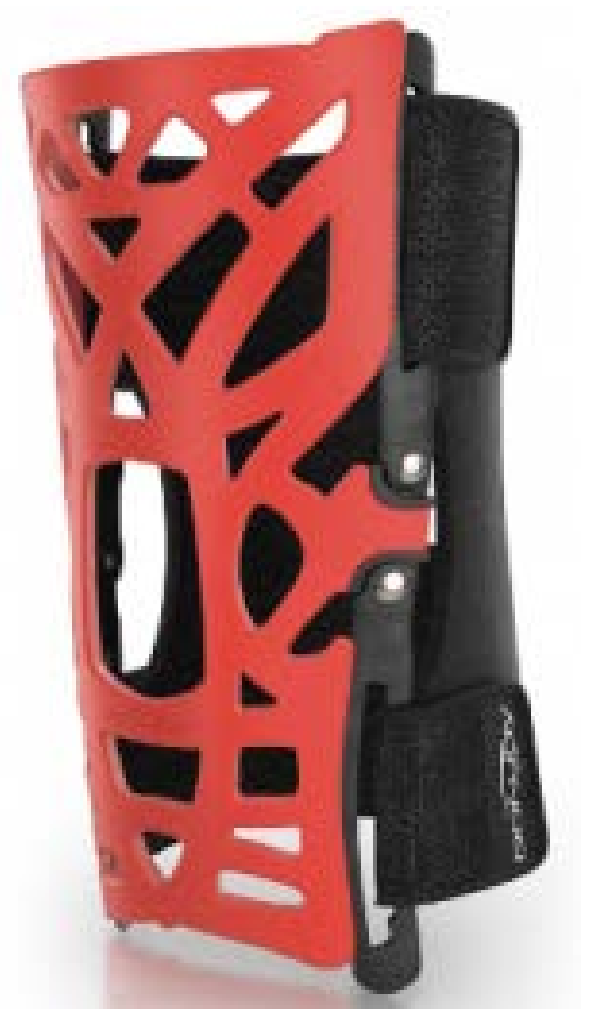

Figure 1. DJO Reaction Brace.

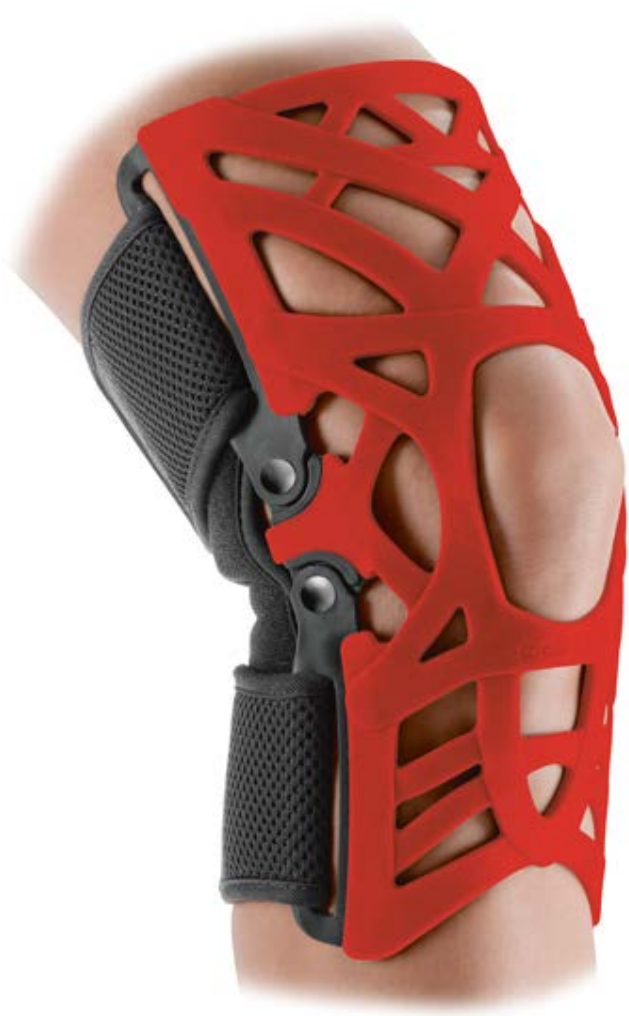

Figure 2. DJO Reaction Brace in position. The silicone elastomeric web design absorbs shock to the patellofemoral joint while the soft silicone condyle pads and cushioned straps maintain its position throughout full range of motion. 


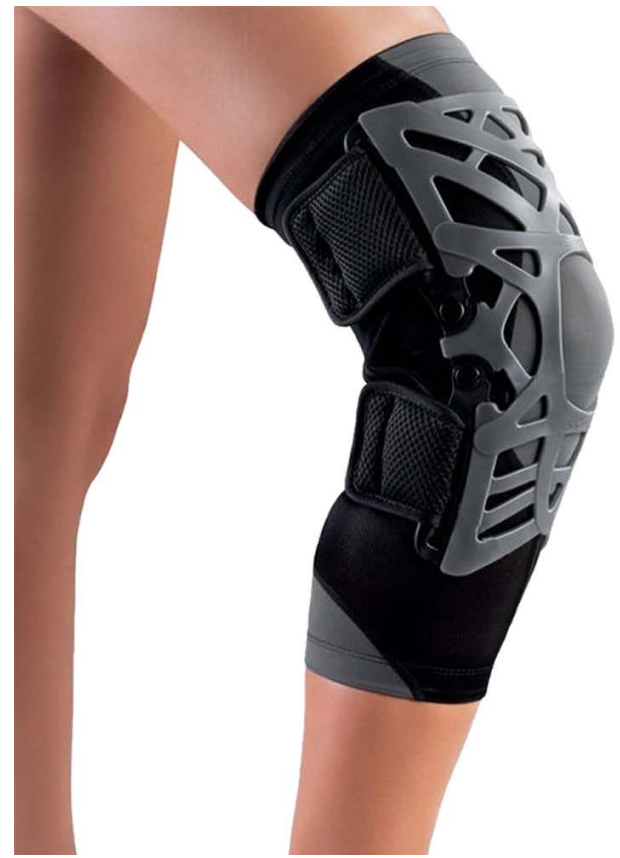

Figure 3. DJO Reaction Brace in position, from the side. Note the knee sleeve donned beneath the knee orthosis for skin protection.

exercise and daily activities that had been limited by pain. Subjects completed baseline measures including the Kujala Anterior Knee Pain Scale (AKPS) and the Knee Injury and Osteoarthritis Outcome Score (KOOS) questionnaire to measure knee function. The AKPS is reliable, valid, and responsive for testing treatment outcomes in patients with patellofemoral pain [10]. Subjects performed a retro-step up test to provide a standardized functional pain score. This test involves standing on a $20 \mathrm{~cm}$ step on the involved, or most painful leg, then lowering the contralateral limb until first the toe and then the heel touches the ground and coming back to a standing position. Patients were asked to perform 20 consecutive repetitions of this test and report their knee pain using a 10 cm visual analog scale (VAS). If patients could not complete 20 repetitions due to pain, loss of balance, prolonged weight-bearing with the foot on the floor, or obvious pushing-off from the floor, then the number of repetitions and the knee pain at that level (using a VAS) was recorded.

\subsection{Statistical Analysis}

Data were recorded and analyzed using Microsoft Excel software.

\section{Results}

Between October 2012 and April 2014, we enrolled 22 subjects that met inclusion and exclusion criteria, five of which were lost to follow-up, leaving 17 subjects available for analysis. Of the five who were lost to follow-up, one elected to drop out because of discomfort with the brace and, although he was offered a refitting and another size if appropriate, he chose to discontinue participation. Consequently, the brace was modified to provide less constrictive posterior knee padding for subsequent patients. Among the 17 subjects, the average time between initial evaluation and the $1^{\text {st }}$ follow-up examination was 55 days. Among the 8 subjects who participated in a second follow-up, the mean time between $1^{\text {st }}$ and $2^{\text {nd }}$ clinical follow-up examinations was 40 days.

Demographic data of the subjects is presented in Table 1 . With an age range of 19 - 39, the average age was 28 years old, and the average duration of symptoms was 15 months. KOOS and Kujala scores from initial evaluation, first, and second clinical follow-ups may be found in Table 2.

\section{Discussion}

While other knees or those knees have been studied for the treatment of patellofemoral pain syndrome, this is 
Table 1. Demographic data.

\begin{tabular}{ccc}
\hline & Male & Female \\
\hline Number & 7 & 10 \\
Age & $24-37$ & $19-39$ \\
Duration of symptoms range (months) & $3-72$ & $6-36$ \\
Duration of symptoms mean (months) & 16 & 16.2 \\
Laterality & & 7 \\
Unilateral & 6 & 5 \\
Right & 4 & 2 \\
Left & 2 & 3 \\
\hline
\end{tabular}

Table 2. KOOS and Kujala scores at initial evaluation, first, and second follow-up examination.

\begin{tabular}{|c|c|c|c|c|c|}
\hline KOOS Score & Initial & First Follow-up & $\begin{array}{l}\text { Second } \\
\text { Follow-up }\end{array}$ & $\begin{array}{l}\text { Relative Improvement, Initial } \\
\text { Exam-1 }{ }^{\text {st }} \text { FU (p-Value) }\end{array}$ & $\begin{array}{l}\text { Relative Improvement } \\
\mathbf{1}^{\text {st }-2^{\text {nd }}}\end{array}$ \\
\hline Symptom & 81 & 85 & 88 & $3.2 \%(\mathrm{p}<0.01)$ & $4.2 \%(\mathrm{p}=0.99)$ \\
\hline Pain & 74.5 & 84 & 85 & $10.7 \%(\mathrm{p}<0.01)$ & $1.4 \%(\mathrm{p}=0.25)$ \\
\hline ADL & 85.8 & 92 & 93 & $6.2 \%(p=0.06)$ & $0.8 \%(p=0.98)$ \\
\hline Sports/Rec & 62.8 & 74 & 86 & $12.9 \%(\mathrm{p}<0.01)$ & $14.3 \%(\mathrm{p}<0.01)$ \\
\hline QOL & 42 & 56 & 59 & $20.2 \%(\mathrm{p}<0.01)$ & $5.2 \%(\mathrm{p}<0.01)$ \\
\hline Kujala Score & 75.6 & 83 & 87 & $9.8 \%(\mathrm{p}<0.01)$ & $5.0 \%(p=0.02)$ \\
\hline
\end{tabular}

the first study of the efficacy and safety of the DJO Reaction knee orthosis. This orthosis is unique in its shockabsorbing elastomeric design, theorized to shift peak loads away from the painful lateral patellofemoral joint, allow improved patellar tracking, and enhance proprioceptive and neurosensory feedback. Findings demonstrated statistically significant improvement within the first 55 days of use in the scoring categories for KOOS Symptoms, Pain, Sports and Recreation, and Quality of life, as well as the Kujala Anterior Knee Pain Scale. Interestingly, the KOOS Quality of Life category displayed the largest improvement during the initial period. While all above measures continued to show improvement between the $1^{\text {st }}$ and $2^{\text {nd }}$ clinical follow-up, only the KOOS Sports and Recreation and Quality of Life and the Kujala Scores demonstrated statistically significant improvement within this time period.

The greatest improvement in objective scores occurred within the initial period, followed by a lesser degree of improvement between the first and second follow-up examinations. This finding may be explained by the immediate biomechanical alteration in patellar tracking imparted by the DJO Reaction knee orthosis. However, the small number of subjects who followed up for a second time (8 of 17) is a limitation. Furthermore, a large proportion of the subjects reported improvement within the first two weeks of wear, along with an adjustment period to the brace within approximately the same time period. The investigators found that appropriate sizing of the DJO Reaction Brace and proper instructions on how to don and adjust the brace were essential components in its tolerance by the subjects and consequently for its routine use during functional activities.

Study limitations include a small sample size of seventeen subjects who completed a follow-up visit. While it may be argued that by failing prior conservative care, including a physical therapy and home exercise program, the patients represent their own control group. This study does not include a true, separate control group. Finally, no direct comparison was made to other available orthoses. Therefore, while statistically and clinically significant improvement was achieved with the DJO Reaction orthosis in these subjects with PFPS, no comparison can be made to other knee orthoses for the treatment of PFPS. Future studies are needed with larger sample sizes to delineate biomechanical risk factors that predict better outcomes with the brace, and to compare among the variety of available knee orthoses in the treatment of PFPS. 


\section{Conclusion}

The DJO Reaction knee orthosis reduces knee pain, increases function, and enhances quality of life in individuals with PFPS. Its unique shock-absorbing elastomeric design likely explains the nearly immediate improvement in symptoms with its use. The majority of functional and symptomatic improvement is achieved within the first two months, followed by continued but lesser improvements in the following period of time. In summary, the DJO Reaction knee orthosis is effective for the conservative treatment of PFPS.

\section{References}

[1] Messier, S.P., Davis, S.E., Curl, W.W., Lowery, R.B. and Pack, R.J. (1991) Etiologic Factors Associated with Patellofemoral Pain in Runners. Medicine and Science in Sports and Exercise, 23, 1008-1015. http://dx.doi.org/10.1249/00005768-199109000-00003

[2] Devereaux, M.D. and Lachmann, S.M. (1984) Patello-Femoral Arthralgia in Athletes Attending a Sports Injury Clinic. British Journal of Sports Medicine, 18, 18-21. http://dx.doi.org/10.1136/bjsm.18.1.18

[3] Dutton, R.A., Khadavi, M.J. and Fredericson, M. (2014) Update on Rehabilitation of Patellofemoral Pain. Current Sports Medicine Reports, 13, 172-178. http://dx.doi.org/10.1249/JSR.0000000000000056

[4] Stathopulu, E, and Baildam, E. (2003) Anterior Knee Pain: A Long-Term Follow-Up. Rheumatology, 42, $380-382$. http://dx.doi.org/10.1093/rheumatology/keg093

[5] Collins, N., Crossley, K., Darnell, R., and Vicenzino, B. (2010) Predictors of Short and Long Term Outcome in Patellofemoral Pain Syndrome: A Prospective Longitudinal Study. BMC Musculoskeletal Disorders, 11, 11. http://dx.doi.org/10.1186/1471-2474-11-11

[6] Draper, C., Jennings, F., Fredericson, M., Besier, T., Gold, G., Beaupre, G. and Delp, S. (2009) Using Real-Time MRI to Quantify Altered Joint Kinematics in Subjects with Patellofemoral Pain and to Evaluate the Effects of a Patellar Brace or Sleeve on Joint Motion. Journal of Orthopedic Research, 27, 571-577. http://dx.doi.org/10.1002/jor.20790

[7] Powers, C.M., Ward, S.R., Chen, Y.J., Chan, L.D. and Terk, M.R. (2004) Effect of Bracing on Patellofemoral Joint Stress while Ascending and Descending Stairs. Clinical Journal of Sport Medicine, 14, 206-214. http://dx.doi.org/10.1097/00042752-200407000-00003

[8] Arazpour, M., Notarki, T.T., Salimi, A., Bani, M.A., Nabavi, H. and Hutchins, S.W. (2013) The Effect of Patellofemoral Bracing on Walking in Individuals with Patellofemoral Pain Syndrome. Prosthetics and Orthotics International, 37, 465-470. http://dx.doi.org/10.1177/0309364613476535

[9] Miller, M.D., Hinkin, D.T. and Wisnowski, J.W. (1997) The Efficacy of Orthotics for Anterior Knee Pain in Military Trainees. A Preliminary Report. The American Journal of Knee Surgery, 10, 10-13.

[10] Wang, D., Morgan, H., Jones, M., Khair, M., and Miniaci, A. (2010) Patient-Reported Outcome Measures for the Knee. The Journal of Knee Surgery, 23, 137-151. http://dx.doi.org/10.1055/s-0030-1268691 